\title{
Realistic antenna modeling for MIMO systems in microcell scenarios
}

\author{
C. Waldschmidt, C. Kuhnert, T. Fügen, and W. Wiesbeck \\ Institut für Höchstfrequenztechnik und Elektronik (IHE), Universität Karlsruhe, Germany
}

\begin{abstract}
This paper shows the potential of MIMO in cellular systems, where small handheld devices are used for the terminals. A complete model of a MIMO communication link is used to integrate accurate antenna modelling into MIMO system simulations. All different effects of mutual coupling between closely spaced antennas are considered. The efficiency or power budget respectively of the antenna arrays in the terminals, which are influenced by mutual coupling effects, is taken into account. Capacity simulation results based on a channel obtained from ray-tracing simulations are shown with cellular phones with up to three Inverted-F antennas.
\end{abstract}

\section{Introduction}

Recent studies have shown the potential of multiple transmit and multiple receive (multiple input multiple output MIMO) antennas to reach high spectral efficiencies, see Winters (1987); Foschini and Gans (1998); Telatar (1998). MIMO systems combine classical techniques such as diversity and beamforming. Additionally they can transmit several datastreams at the same time and frequency in parallel. The capacity or spectral efficiency respectively depends on the signal to noise ratio (SNR) and the correlation properties among the channel transfer functions of different pairs of transmit and receive antennas. Uncorrelated channel transfer functions or channel coefficients in the flat fading case lead to high capacities for MIMO systems, that are based on multiplexing and use several subchannels, Chuah et al. (2002).

Many handheld devices like laptops or palmtops require small antenna spacings. This requirement is contrary to uncorrelated channel coefficients for MIMO systems based on space diversity, thus other diversity techniques have to be applied.

In almost all studies about MIMO, the SNR is assumed to be independent of the correlation properties of the channel

Correspondence to: $\mathrm{C}$. Waldschmidt

(christian.waldschmidt@ihe.uka.de) matrix $\mathbf{H}$, which contains the channel coefficients. Especially for small antenna spacings this assumption does not hold, as mutual coupling influences both SNR and $\mathbf{H}$. In recent studies about mutual coupling this effect has been neglected, see Svantesson (2001), Stoytchev et al. (2001) and Waldschmidt et al. (2002). The same holds for mismatching of the antenna arrays. For MIMO systems with a constant transmit power the SNR is reduced for small antenna spacings, since the effective gain of the antennas is reduced, which decreases the capacity.

For compact antenna arrays it is necessary to analyze the SNR and the properties of $\mathbf{H}$ together. Therefore an extended channel, including transmitter (Tx), receiver ( $\mathrm{Rx})$, antennas and the physical channel is taken into account. The model of the whole system allows to analyze the whole radio frequency transmission chain, including mutual coupling and mismatching effects.

This paper is organized as follows. In Sect. 2 the system model, including an accurate way to model the antennas, is shown. In Sects. 3 and 4 the capacity calculation of a MIMO system and power considerations of the link are given. Sections 5 to 7 present an example of a MIMO system in a microcell scenario with realistic base station antennas and a small handheld device with up to three antennas. The correlation properties as well as the capacity and the power gain of this example are discussed in detail. Section 8 draws a conclusion.

\section{System model}

The complete radio frequency transmission chain consists of 5 elements: signal source (transmitter) - transmit antennas physical channel - receive antennas - signal drain (receiver), given in Fig. 1. The number of transmit antennas is $M$, the one of receive antennas $N$. The single components are modelled as networks which are described by scattering matrices, see e.g. Pozar (1998). 


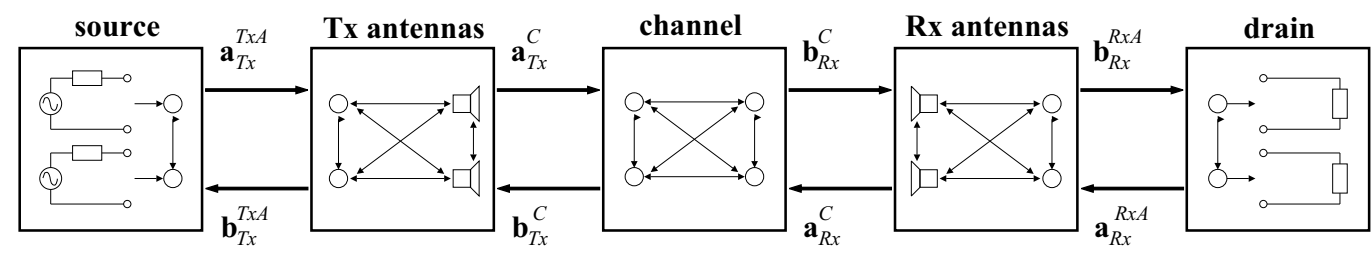

Fig. 1. System model of the complete radio frequency transmission chain. All elements are described by scattering matrices.

\subsection{Signal source}

The signal source is the beginning of the transmission chain and determines the power distribution among the transmit antennas. It has $M$ ports, according to the number of transmit antennas. The output impedances of the signal source are characterized by the reflection matrix $\mathbf{r}_{T x}^{S}$, where the upper index $S$ denotes source. The outward propagating wave vector is

$\boldsymbol{b}_{T x}^{S}=\boldsymbol{b}_{0, S}^{S}+\mathbf{r}_{T x}^{S} \boldsymbol{a}_{T x}^{S}$.

$\boldsymbol{b}_{0, S}^{S}$ is the wave vector fed into the network, which determines the power distribution among the source ports. $\boldsymbol{a}_{T x}^{S}$ is the power reflected by the transmit antennas.

\subsection{Transmit antennas}

The antennas suffer from different effects when placed close together. They couple and interact, and cannot be considered as independent elements. The main effects, all of which have to be taken into account, are:

- The shape of the radiation pattern of the single antennas changes due to the other antennas. If e.g. omnidirectional antennas are used a pattern diversity effect occurs.

- The active gain of the single antennas has to be considered. The active gain is defined as the gain of a single antenna surrounded by the other terminated antennas. As the other antennas influence the pattern, the gain in terms of directivity changes, accordingly it is called the active gain. Additionally, the energy radiated from an antenna may be directly absorbed by another closely spaced antenna, see Kraus (1988), thus the active gain is reduced. With this definition the active gain is a property of each individual antenna in an array and depends on the array topology and termination of the antennas. It is not a function of the incident field.

- The signals received or transmitted by an antenna directly couple to the other antennas, only amplitude and phase shifted.

All these effects are included in the general scattering parameter description of the transmit antennas based on Wiesbeck and Heidrich (1998)

$$
\left(\begin{array}{l}
\boldsymbol{b}_{T x A}^{T x A} \\
\boldsymbol{b}_{F}^{T x A}
\end{array}\right)=\left(\begin{array}{cc}
\mathbf{S}_{T x T x}^{T x A} & \mathbf{S}_{T x F}^{T x A} \\
\mathbf{S}_{F T x}^{T x A} & \mathbf{S}_{F F}^{T x A}
\end{array}\right)\left(\begin{array}{c}
\boldsymbol{a}_{T x A}^{T x A} \\
\boldsymbol{a}_{F}^{T x A}
\end{array}\right)
$$

where the upper index $T x A$ denotes transmit antennas. Each antenna in the array is basically seen as a two port network. One port describes the excitation side of the antenna whereas the other port describes the far field properties. The submatrix $\mathbf{S}_{T x T x}^{T x A}$ describes the excitation ports of the antennas. It contains the scattering parameters corresponding to the self (diagonal elements) and mutual coupling (off-diagonal elements) impedances of the array Kraus (1988). The self impedance of an antenna is the input impedance if the antenna is remote in other words isolated from its surrounding. With other antennas in the near surrounding of an antenna the influence of these antennas on the input impedance is considered. The mutual coupling impedances describe the coupling between the antennas. The submatrices $\mathbf{S}_{F T x}^{T x A}$ and $\mathbf{S}_{T x F}^{T x A}$ describe the transmission of the signals from the excitation side of the antenna network to the far field and vice versa, thus contain information on the pattern and gain of the antennas. They are reciprocal. The submatrix $\mathbf{S}_{F F}^{T x A}$ contains the structural antenna scattering of the array with the excitation ports of the antennas terminated in matched loads. The elements of the structural antenna scattering matrix and the transmission matrices are directional, i.e. they are a function of the geometry and change for different angles of arrival and departure for different paths.

\subsection{Physical channel}

The channel is modelled by

$$
\left(\begin{array}{c}
\boldsymbol{b}_{T x}^{C} \\
\boldsymbol{b}_{R x}^{C}
\end{array}\right)=\left(\begin{array}{cc}
\mathbf{S}_{T x}^{C} & \mathbf{S}_{T x R x}^{C} \\
\mathbf{S}_{R x T x}^{C} & \mathbf{S}_{R x}^{C}
\end{array}\right)\left(\begin{array}{c}
\boldsymbol{a}_{T x}^{C} \\
\boldsymbol{a}_{R x}^{C}
\end{array}\right),
$$

where the upper index $C$ denotes channel. This scattering matrix expresses the relationship between the far field ports of the transmit and receive antenna arrays thus the coupling disappears by definition. Additionally it is assumed that there is no reflection from the far field. In other words nothing of the once radiated transmit power is received by the transmit antennas, thus the submatrices $\mathbf{S}_{T x}^{C}$ and $\mathbf{S}_{R x}^{C}$ equal the zero matrix.

\subsection{Receive antennas and signal drain}

The receive antennas are described analogue to the transmit antennas written with the upper index $R x A$. For the sake of completeness the mathematical description is given here

$\left(\begin{array}{l}\boldsymbol{b}_{F}^{R x A} \\ \boldsymbol{b}_{R x}^{R x A}\end{array}\right)=\left(\begin{array}{cc}\mathbf{S}_{F F}^{R x A} & \mathbf{S}_{F R x}^{R x A} \\ \mathbf{S}_{R x F}^{R x A} & \mathbf{S}_{R x R x}^{R x A}\end{array}\right)\left(\begin{array}{l}\boldsymbol{a}_{F}^{R x A} \\ \boldsymbol{a}_{R x}^{R x A}\end{array}\right)$. 
The signal drain is similar to the signal source. It is described by the reflection matrix $\mathbf{r}_{R x}^{D}$ with the dimension $N \times N$, where the upper index $D$ denotes drain.

\subsection{Merging of inner components}

The five units given in the previous section are connected together now. We proceed in two steps. First, the inner three components i.e. transmit antennas, physical channel and the receive antennas are merged. The unilateral channel is introduced, which simplifies the problem. Second, the signal source and signal drain are connected to the network. After that the channel matrix $\mathbf{H}^{\text {ext }}$ of the extended channel with signal source and drain is given, which allows for capacity calculation.

The transmit antenna scattering matrix is cascade connected with the scattering matrix of the physical channel. These matrices are then connected with the scattering matrices of the receive antennas, which yields

$$
\begin{aligned}
\mathbf{S}_{T x T x}^{H}= & \mathbf{S}_{T x T x}^{T x A}+\mathbf{S}_{T x F}^{T x A} \mathbf{S}_{T x R x}^{C} \\
\cdot & \left(\mathbf{I}-\mathbf{S}_{F F}^{R x A} \mathbf{S}_{R x T x}^{C} \mathbf{S}_{F F}^{T x A} \mathbf{S}_{T x R x}^{C}\right)^{-1} \\
\cdot & \mathbf{S}_{F F}^{R x A} \mathbf{S}_{R x T x}^{C} \mathbf{S}_{F T x}^{T x A} \\
\mathbf{S}_{T x R x}^{H}= & \mathbf{S}_{T x F}^{T x A} \mathbf{S}_{T x R x}^{C} \\
\cdot & \left(\mathbf{S}-\mathbf{S}_{F F}^{R x A} \mathbf{S}_{R x T x}^{C} \mathbf{S}_{F F}^{T x A} \mathbf{S}_{T x R x}^{C}\right)^{-1} \cdot \mathbf{S}_{F R x}^{R x A} \\
\mathbf{S}_{R x T x}^{H}= & \mathbf{S}_{R x F}^{R x A} \\
& \cdot\left(\mathbf{I}-\mathbf{S}_{R x T x}^{C} \mathbf{S}_{F F}^{T x A} \mathbf{S}_{T x R x}^{C} \mathbf{S}_{F F}^{R x A}\right)^{-1} \\
& \cdot \mathbf{S}_{R x T x}^{C} \mathbf{S}_{F T x}^{T x A} \\
\mathbf{S}_{R x R x}^{H}= & \mathbf{S}_{R x R x}^{R x A}+\mathbf{S}_{R x F}^{R x A} \\
\cdot & \left(\mathbf{I}-\mathbf{S}_{R x T x}^{C} \mathbf{S}_{F F}^{T x A} \mathbf{S}_{T x R x}^{C} \mathbf{S}_{F F}^{R x A}\right)^{-1} \\
\cdot & \mathbf{S}_{R x T x}^{C} \mathbf{S}_{F F}^{T x A} \mathbf{S}_{T x R x}^{C} \mathbf{S}_{F R x}^{R x A} .
\end{aligned}
$$

Equations (5) to (9) describe the extended channel without signal source and drain

$\mathbf{S}^{H}=\left(\begin{array}{ll}\mathbf{S}_{T x T x}^{H} & \mathbf{S}_{T x R x}^{H} \\ \mathbf{S}_{R x T x}^{H} & \mathbf{S}_{R x R x}^{H}\end{array}\right)$.

The back transmission of signals through the physical channel is subject to the channel attenuation, thus the power reradiated by the receiver and received by the transmitter is twice as strong attenuated as the signals at the receiver. Therefore it is justified to neglect to back transmission, see also Wallace and Jensen (2002), and to set $\mathbf{S}_{T x R x}^{C}=\mathbf{0}$, which simplifies Eqs. (5) to (9). We call this channel unilateral channel. The result is

$\mathbf{S}^{H}=\left(\begin{array}{cc}\mathbf{S}_{T x T x}^{T x A} & \mathbf{0} \\ \mathbf{S}_{R x F}^{R x A} \mathbf{S}_{R x T x}^{C} \mathbf{S}_{F T x}^{T x A} \mathbf{S}_{R x R x}^{R x A}\end{array}\right)$.

The term $\mathbf{S}_{R x F}^{R x A} \mathbf{S}_{R x T x}^{C} \mathbf{S}_{F T x}^{T x A}$ in Eq. (10) describes the transmission of the signals from the input ports of the transmit antennas to the output ports of the receive antennas. Using the

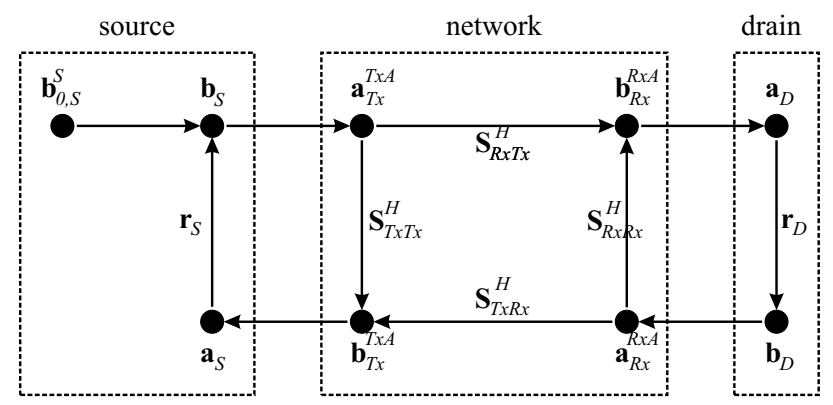

Fig. 2. Signal flow graph for the network above.

Heavyside transformation Pozar (1998), it can be expressed as the ratio of the voltages $U$ at the transmit $m$ and receive $n$ antennas.

$S_{R x T x, n m}^{H}=\left.\sqrt{\frac{Z_{0, m}}{Z_{0, n}}} \frac{U_{n}}{U_{m}}\right|_{a_{k}=0} \quad$ for all $\quad k \neq m$,

where $Z_{0}$ is the characteristic impedance of the scattering parameters.

\subsection{Termination with source and drain}

Since the mutual coupling effects strongly depend on the termination of the transmit and receive antennas, the signal source and drain are added to the inner components of the network. Figure 2 shows the merged inner components network terminated with source and drain. With the wave vectors $\boldsymbol{a}_{T x}^{T x A}, \boldsymbol{a}_{R x}^{R x A}, \boldsymbol{b}_{T x}^{T x A}$, and $\boldsymbol{b}_{R x}^{R x A}$ at the input and output ports of the terminated network is is possible to calculate the power gain of the extended channel and the extended channel matrix $\mathbf{H}^{\text {ext }}$. Some basic scattering parameter calculations applied to the network consisting of the merged inner components (Eq. 10) and the signal source and drain yields

$$
\begin{aligned}
\boldsymbol{a}_{T x}^{T x A}= & \left(I-\mathbf{r}_{T x}^{S} \mathbf{S}_{T x T x}^{H}\right)^{-1} \boldsymbol{b}_{0, S}^{S} \\
\boldsymbol{a}_{R x}^{R x A}= & \left(I-\mathbf{r}_{R x}^{D} \mathbf{S}_{R x R x}^{H}\right)^{-1} \mathbf{r}_{R x}^{D} \mathbf{S}_{R x T x}^{H} \\
& \cdot\left(I-\mathbf{r}_{T x}^{S} \mathbf{S}_{T x T x}^{H}\right)^{-1} \boldsymbol{b}_{0, S}^{S} \\
\boldsymbol{b}_{T x}^{T x A}= & \left(I-\mathbf{S}_{T x T x}^{H} \mathbf{r}_{T x}^{S}\right)^{-1} \mathbf{S}_{T x T x}^{H} \boldsymbol{b}_{0, S}^{S} \\
\boldsymbol{b}_{R x}^{R x A}= & \left(I-\mathbf{S}_{R x R x}^{H} \mathbf{r}_{R x}^{D}\right)^{-1} \\
& \cdot\left(\mathbf{S}_{R x T x}^{H}+\mathbf{S}_{R x T x}^{H} \mathbf{r}_{T x}^{S}\left(I-\mathbf{S}_{T x T x}^{H} \mathbf{r}_{T x}^{S}\right)^{-1} \mathbf{S}_{T x T x}^{H}\right) \\
& \cdot \boldsymbol{b}_{0, S}^{S} .
\end{aligned}
$$

The extended channel matrix $\mathbf{H}^{\text {ext }}$, which allows for the capacity calculations in the following section, expresses the ratio of the voltages at the receive antennas $\boldsymbol{U}_{R x}$ to the voltages at the transmit antennas $\boldsymbol{U}_{T x}$.

$\boldsymbol{U}_{R x}=\mathbf{H}^{e x t} \boldsymbol{U}_{T x}$. 


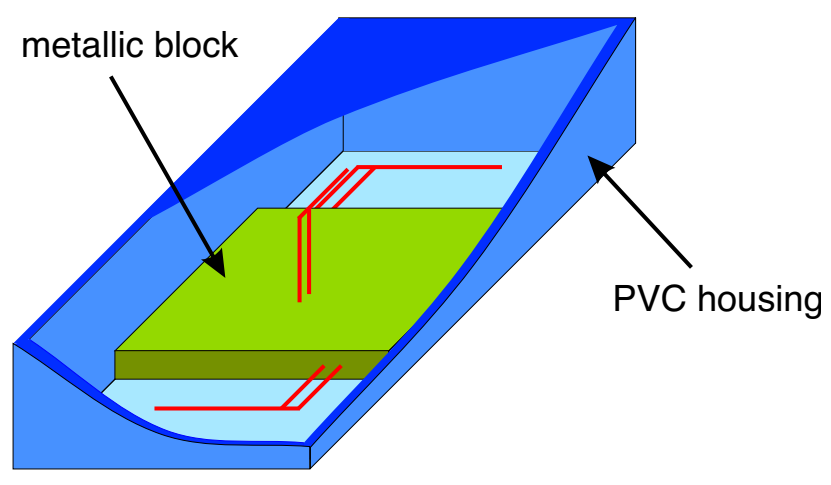

Fig. 3. Cellular phone model with three Inverted-F antennas. The size of the housing is $4.3 \times 10.5 \times 2.7 \mathrm{~cm}^{3}$.

Using the Heavyside transformation leads to

$Z_{0, n}^{1 / 2}\left(\boldsymbol{a}_{R x}^{R x A}+\boldsymbol{b}_{R x}^{R x T}\right)=\mathbf{H}^{e x t} Z_{0, m}^{1 / 2}\left(\boldsymbol{a}_{T x}^{T x A}+\boldsymbol{b}_{T x}^{T x A}\right)$.

To solve this equation for $\mathbf{H}^{\text {ext }}$ the voltages $\boldsymbol{U}_{R x}$ can be expressed as a function of the voltages $\boldsymbol{U}_{T x}$. By using Eqs. (12-16) one yields

$$
\begin{aligned}
\mathbf{H}^{e x t} & =\left(Z_{0, n}\right)^{1 / 2}\left(\mathbf{I}+\mathbf{r}_{R x}^{D}\right)\left(\mathbf{I}-\mathbf{S}_{R x R x}^{H} \mathbf{r}_{R x}^{D}\right)^{-1} \\
& \cdot \mathbf{S}_{R x T x}^{H}\left(\mathbf{I}+\mathbf{S}_{T x T x}^{H}\right)^{-1}\left(Z_{0, m}\right)^{1 / 2} .
\end{aligned}
$$

\section{Capacity calculations}

The instantaneous capacity of MIMO transmission channels in the presence of spatially uncolored gaussian distributed noise can be calculated by ${ }^{1}$

$C=\log _{2}\left(\operatorname{det}\left(I+\frac{S N R}{n} \mathbf{H H}^{\dagger}\right)\right)$

In order to assess a MIMO transmission system by the capacity, usually a fixed $S N R$ (see e.g. Jensen, 2001) and a channel matrix, normalized with $\|\mathbf{H}\|^{2}=n_{T x} n_{R x}$ is used. $\|\mathbf{H}\|$ denotes the Frobenius Norm of $\mathbf{H}$. But if comparing different antennas in the same scenario, a fixed SNR and a normalized channel matrix any interrelation between the mean attenuation and the correlation properties of $\mathbf{H}$, that strongly influence the capacity and that are both influenced by mutual coupling as shown before, is neglected. If $\mathbf{H}$ is not normalized with the Frobenius Norm, that means the path loss and the gain of single antenna elements are included in $\mathbf{H}$, (19) can be written as

$C=\log _{2}\left(\operatorname{det}\left(I+\frac{P_{T}}{\sigma^{2} n} \mathbf{H H}^{\dagger}\right)\right)$.

This formulation allows for a fair comparison of different antenna arrays, since both the correlation properties of $\mathbf{H}$ and

\footnotetext{
${ }^{1} I$ denotes the identity matrix $n=\min (n, m) \dagger$ denotes conjugate complex transpose
}

the power budget of the link, i.e. the efficiency of the arrays, is taken into account. $P_{T}$ is the total transmit power which is equally distributed among all transmit antennas, if no channel state information at the transmitter is available. If the channel is known at the receiver, the capacity can be increased by using waterfilling, see Bach-Andersen (2000). For the capacity calculations given in the following, $\mathbf{H}^{\text {ext }}$ of Eq. (18) is used in Eq. (20) and no channel state information at the transmitter is assumed.

\section{Power considerations}

In order to assess the efficiency in term of power of an array in a MIMO system, we use the transmission gain of the whole MIMO system model. The power gain $G_{H^{e x t}}$ of the extended channel is the ratio of the real power delivered to the signal drain to the real power fed into the transmit antennas. It allows to draw conclusions on the efficiency of the whole transmission chain.

$G_{H^{e x t}}=\frac{\boldsymbol{b}_{R x}^{R x A \dagger} \boldsymbol{b}_{R x}^{R x A}-\boldsymbol{a}_{R x}^{R x A \dagger} \boldsymbol{a}_{R x}^{R x A}}{\boldsymbol{a}_{T x}^{T x A^{\dagger}} \boldsymbol{a}_{T x}^{T x A}-\boldsymbol{b}_{T x}^{T x A \dagger} \boldsymbol{b}_{T x}^{T x A}}$.

Using this definition the power gain depends on the power distribution among the elements of the excitation vector $\boldsymbol{b}_{0, S}^{S}$. Note, that the power fed into the transmit antennas is not equal to the radiated power, since there occur losses in the transmit antennas due to the losses in the single antennas and due to mutual coupling effects. Since in MIMO system simulations or measurements a large number of different channel realizations is considered the power gain of the extended channel $G_{H^{e x t}}$ is a random variable.

In the following an example showing the application of the network model is given. We consider a MIMO link between a small handheld device and a base station in a microcell scenario.

\section{Antennas in the handheld device}

For the handheld devices half-wavelength dipole antennas and Inverted-F antennas were used. The aim of the antenna setup was to combine different diversity techniques, such as pattern, spatial and polarization diversity, to obtain uncorrelated signals at the antennas, see Waldschmidt et al. (2003). Additionally polarization diversity makes the antenna system robust against polarization mismatching. The simulation model of the handheld device consists of a metallic block, representing the battery and the display, and a PVC housing with a wall thickness of $2 \mathrm{~mm}$. The size of the housing is $4.3 \times 10.5 \times 2.7 \mathrm{~cm}^{3}$. Three different antenna configurations in the handheld device were modelled: a) two crossed dipoles, see Fig. 4, b) 3 Inverted-F antennas at a large (length $=5 \mathrm{~cm}$ ) and c) at a small (length $=3 \mathrm{~cm}$ ) metallic block, see Fig. 3. Usually Inverted-F antennas require a large ground plane, which is not given in the small handheld device. Thus the metallic block, representing the ground plane, 


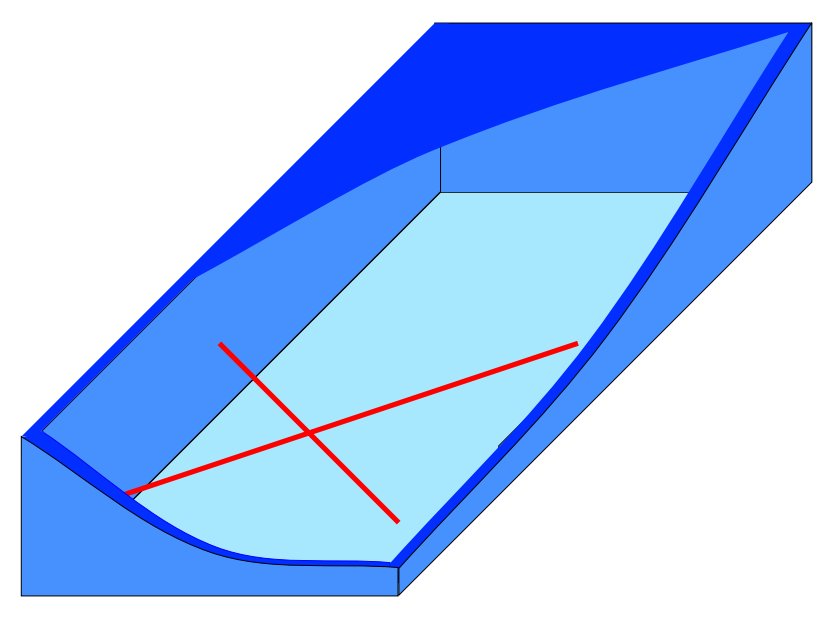

Fig. 4. Cellular phone model with 2 crossed dipole antennas.

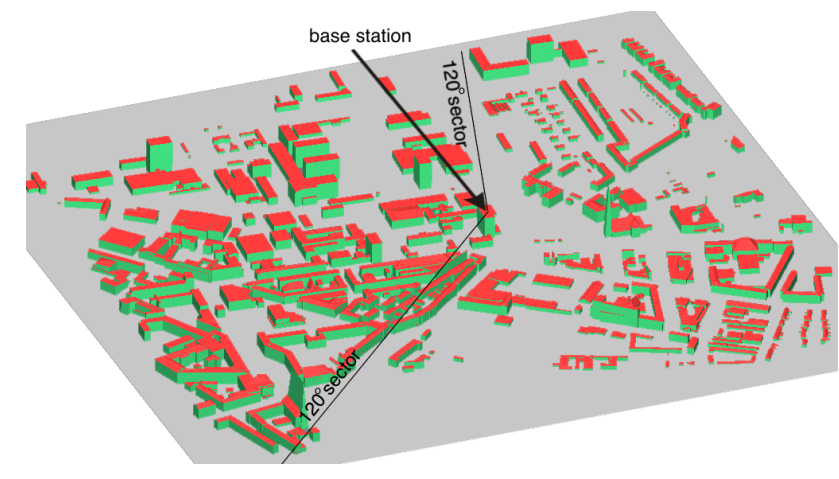

Fig. 5. Microcell scenario for the ray-tracing simulations.

acts as a part of the antenna and influences the system performance. Due to that reason, the size of the metallic block changes the pattern of the Inverted-F antennas compared to an idealized antenna and the mutual coupling impedances among the antennas. All antenna configurations were simulated with FEKO, see (www.feko.com, 2003), a standard EM-code to calculate the pattern of the coupled system and the self- and mutual coupling impedance matrix. For the system simulations, self impedance matching between the antennas and the loads (signal source and drain) was assumed. In other words, the single antennas were perfectly matched, but the mutual coupling impedances were open circuited. Perfect (in terms of power) conjugate complex matching is very unrealistic for handheld devices, since the mutual coupling impedances can hardly be matched. They change during usage of the device.

\section{Microcell scenario}

In the following scenario a link between a small handheld device and a base station in a microcell scenario is considered. The focus here is on the antennas in the handheld device and the base station, thus no interference or multiple users are assumed.

The base station is equipped with three antennas with spacings of 1 meter. The pattern of the commercially available base station antenna "Kathrein Antenne 735147 (GSM 1800)" was modelled, see Baldauf et al. (2001), for the simulations. The channel data were obtained from ray-tracing simulations of a microcell scenario. The campus of the University of Karlsruhe and the surrounding buildings were used for the simulations, see Fig. 5. The base station with three Kathrein antennas radiating into the same $120^{\circ}$ sector, is placed on one of the highest buildings in the scenario, $3 \mathrm{~m}$ above the roof. At 2000 randomly distributed locations on the campus the channel data are collected. The power azimuth spectrum at the base station is relatively narrow, since there are no scatterers around the antennas. At the mobile the angular spread is wide, as the mobile is surrounded by scatterers. The channel data are normalized to obtain a constant mean attenuation of the link. That means, the incoherent sum of the path weights $\Gamma$ of all paths $v$ and polarizations of one channel realization are added and set constant.

const $=\sum_{\nu=1}^{P} \Gamma_{\vartheta \vartheta}^{v}+\Gamma_{\vartheta \psi}^{v}+\Gamma_{\psi \vartheta}^{v}+\Gamma_{\psi \psi}^{v}$,

where the subscript denotes the polarization at transmitter and receiver of the path $\nu . P$ is the total number of paths. This normalization removes the slow fading effects, but it does not influence the (spatial-) fast fading, which is essential for MIMO. Using the Frobenius Norm to normalize the channel matrices, as often done in MIMO system simulations, would change the probability distribution of the power of the fast fading signal. The normalized channel data serve as an input for the network model. The antennas are considered separately in the network, thus the power budget of the link and the efficiency of the antennas is fully taken into account.

For comparison, the same simulation was performed without complete handheld devices, but with arrays consisting of parallel $\lambda / 2$-dipoles with one wavelength spacing. Thus uncorrelated channel coefficients can be expected, resulting in a optimum MIMO system.

\section{Simulation results}

Table 1 shows the results. In the second column the power correlation coefficient among the signals at the handheld device are given. The third column shows the $10 \%$ outage capacity in bit/s/Hz for a given constant transmit power. The transmit power was chosen to result in a $10 \%$ outage capacity for the SISO system of $3.5 \mathrm{bit} / \mathrm{s} / \mathrm{Hz}$. In the last column the ratio of the mean transmission gain of the MIMO system to the mean transmission gain of a SISO system with one half-wavelength dipole at the transmitter and at the receiver is given. The MIMO systems with one wavelength antenna spacings and no influence of any housing etc. perform best, as shown in the second and third row of Table 1. They can 
Table 1. Simulation results.

\begin{tabular}{lccc}
\hline $\begin{array}{l}\text { antenna } \\
\text { configuration }\end{array}$ & $\begin{array}{c}\text { power } \\
\text { correlation } \\
\text { at mobile }\end{array}$ & $\begin{array}{c}10 \% \text { outage } \\
\text { capacity } \\
\text { in } \frac{\text { bit }}{\text { sHz }}\end{array}$ & $\begin{array}{c}G_{H} / G_{S I S O} \\
\text { at } 50 \% \\
\text { in } \mathrm{dB}\end{array}$ \\
\hline 2 dipoles, $\lambda$ spacing & $\leq 0.1$ & 12,9 & 8 \\
3 dipoles, $\lambda$ spacing & $\leq 0.1$ & 14.5 & 11 \\
& & & \\
2 crossed dipoles & $\leq 0.1$ & 11.5 & 6.9 \\
3 Inv.-F, large block & $\leq 0.35$ & 13 & 9.6 \\
3 Inv.-F, small block & $\leq 0.2$ & 13.5 & 9.6 \\
SISO & - & 3.5 & 0 \\
\hline
\end{tabular}

be considered as a reference for the other antenna configurations in the handheld device for a comparison of MIMO systems with equal number of antennas. It is evident, that all small antenna arrays in the handheld device perform well in terms of correlation and power and thus lead to capable MIMO systems. Surprisingly the handheld device with the large metallic block does not outperform the one with the small block, though the antenna spacings are larger. This is due to the fact, that the pattern diversity between the antennas is stronger for the small metallic block. That clearly shows, that the whole configuration of the handheld device has to be taken into account.

\section{Conclusion}

The paper shows how to introduce detailed antenna modeling into MIMO system simulations, when using compact antenna arrays, that suffer from mutual coupling. Several antenna array configurations, that fit into small handheld devices are presented and discussed in detail. For the assessment of small antenna arrays it is necessary to consider both first the correlation properties of the signals and second the efficiency in terms of power of the array. It is shown, that MIMO works well with small antenna array configurations, when exploiting combinations of different diversity techniques such as polarization, pattern and spatial diversity. This framework allows for the examination of the behavior of compact arrays in MIMO system simulations.

\section{References}

Bach-Andersen, J.: Array gain and capacity for known random channels with multiple element arrays at both ends, IEEE Journal on Selected Areas in Communications, 18, 2172-2178, 2000.

Baldauf, M., Herschlein, A., and Wiesbeck, W.: Schutzabstände in der Mobilkommunikation, Frequenz, 55, 310-316, 2001.

Chuah, C., Tse, D., Kahn, J., and Valenzuela, R.: Capacity Scaling in MIMO Wireless Systems Under Correlated Fading, IEEE Transactions on Information Theory, 48, 637-650, 2002.

Foschini, G. and Gans, M.: On limits of wireless communications in a fading environment when using multiple antennas, Wireless Personal Communications, 6, 311-335, 1998.

Jensen, M.: Characteristics of measured 4x4 and 10x10 MIMO wireless channel data at $2.4 \mathrm{GHz}$, IEEE International Symposium on Antennas and Propagation, 3, 95-98, 2001.

Kraus, J.: Antennas, Electrical Engineering Series, McGraw-Hill International Editions, second edn., 1988.

Pozar, D. M.: Microwave Engineering, John Wiley \& Sons, Inc., 1998.

Stoytchev, M., Safar, H., Moustakas, A. L., and Simon, S.: Compact antenna arrays for MIMO applications, IEEE International Symposium on Antennas and Propagation, 3, 708-711, 2001.

Svantesson, T.: Mutual coupling effects on the capacity of multielement antenna systems, IEEE Proceedings of Acoustics, Speech, and Signal Processing Conference, 2, 2485-2488, 2001.

Telatar, I.: Capacity of Multi-Antenna Gaussian Channels, Technical Memorandum, Bell Laboratories, Lucent Technologies, October 1995, then published in European Transactions on Telecommunications, 10, 585-595, 1998.

Waldschmidt, C., v. Hagen, J., and Wiesbeck, W.: Influence and Modelling of Mutual Coupling in MIMO and Diversity Systems, IEEE International Symposium on Antennas and Propagation AP-S, San Antonio, Texas, 3, 190-193, 2002.

Waldschmidt, C., Kuhnert, C., Schulteis, S., and Wiesbeck, W.: Compact MIMO arrays based in Polarization Diversity, IEEE internatinal Symposium on Antennas and Propagation, Columbus, Ohio, pp. 499-502, 2003.

Wallace, J. W. and Jensen, M. A.: The capacity of MIMO wireless systems with mutual coupling, IEEE Proceedings of the Vehicular Technology Conference, 2, 696-700, 2002.

Wiesbeck, W. and Heidrich, E.: Wide-band multiport antenna characteristics by polarimetric RCS measurements, IEEE Transactions on Antennas and Propagation, 46, 314-350, 1998.

Winters, J. H.: On the Capacity of Radio Communication Systems with Diversity in a Rayleigh Fading Environment, IEEE Journal on Selected Areas in Communications, 5, 871-877, 1987. 